\title{
Versatile high repetition rate 2 micron pulsed source based on wideband parametric conversion
}

\author{
Adrien Billat, Steevy Cordette, and Camille-Sophie Brès, Member, IEEE
}

\begin{abstract}
We report an all-fiber pulsed source based on parametric conversion followed by thulium amplification able to deliver picosecond pulses at a repetition rate selectable between 2 and $5 \mathrm{GHz}$, of which central wavelength can be freely selected in the 2 micron region. A very versatile Nyquist pulse shaping of the parametric pump, which allows for the electrical control of the pulse train, enables such a freedom in the repetition rate selection, as well as some control in the pulse duration. We also show that data can be embedded in the output pulse train resulting in a high quality $\mathrm{Gb} / \mathrm{s}$ return-to-zero transmitter. Such a programmable short-wave infrared laser is of high interest for sensing or nonlinear optics applications around $2000 \mathrm{~nm}$ that require a fine adjustment in both the spectral and temporal domains.
\end{abstract}

Index Terms-Doped fiber amplifiers, fiber lasers, fiber nonlinear optics, optical pulse generation, optical wavelength conversion.

\section{INTRODUCTION}

Pulsed lasers operating in the $2 \mu \mathrm{m}$ spectral region are now widely used in various applications, ranging from transparent plastic material processing, laser lithotripsy or tissue ablation [1], remote sensing and lidars [2], advanced communication research [3], or for driving nonlinear light generation deeper in the mid-IR, where sub-ns pulse durations and high peak powers are required [4], [5]. Compelled by these demands, substantial progress has recently been made in thulium-doped and holmium-doped lasers and amplifiers. Nonetheless even if modelocked thulium-doped fiber (TDF) lasers are the widespread solution to address these needs, their cavity length generally prevents output repetition rates to be higher than a few tens of MHz. For a subset of applications, a higher and programmable repetition rate is of interest. Being able to choose the duty cycle of the source would allow for the control of the pulse peak power, a particularly attractive

Manuscript received August 26, 2015; revised November 16, 2015, accepted November 20, 2015.

This work was supported by the European Research Council under grant agreement ERC-2012- StG 306630-MATISSE.

All authors are with the Photonic Systems Laboratory, Institute of Electrical Engineering, Ecole Polytechnique Federale de Lausanne (EPFL), Lausanne, Switzerland (e-mail: adrien.billat@epfl.ch). Copyright (c) 2015 IEEE. Personal use of this material is permitted. However, permission to use this material for any other purposes must be obtained from the IEEE by sending a request to pubs-permissions@ieee.org. feature for driving cascaded nonlinear effects towards the mid-IR. A high repetition rate cavity less pulsed source could also be combined with modulation capability for 2 micron communication. In addition, modelocked TDF laser cavities generally do not contain any optical filtering element such that the laser wavelength is rarely tunable. A wavelength tunable modelocked 2 micron laser was demonstrated but with $\mathrm{MHz}$ repetition rate [6]. The difficulty to tune the wavelength can be a significant limitation for absorption line spectroscopy or nonlinear optics experiments, where one must achieve phase matching in a dispersive medium. On the other hand bulk solid state thulium lasers do not reach the performances of fiber sources in terms of ruggedness, mechanical stability and ease of operation.

In this work we demonstrate an all-fiber 2 micron wavelength tunable high repetition ration rate pulsed source. The fiber laser design is based on parametric wavelength conversion using exclusively standard telecom components, apart from the gain media, a concept demonstrated a few years ago [7]. The core operating principle relies on the efficient light conversion from a highly reconfigurable telecom pulsed source toward the short-wave infrared (SWIR) [8]. The remainder of the paper is organized as follows. This operating principle and the source implementation are described in section II. In section III, the experimental characterization of the pulsed parametric converter and the tunable 2 micron pulsed source is presented. In addition, we also show that the source can be adapted as a 2 micron transmitter. Finally the conclusions and discussion are given in section IV.

\section{PRINCIPLE AND EXPERIMENTAL SETUP}

\section{A. Operating principle}

In the presented scheme, light generation around 2 micron is initiated by parametric conversion in a highly nonlinear fiber (HNLF). A tunable signal wave in the O-band (1250$1350 \mathrm{~nm}$ ) interacts with an L-band pump via degenerate fourwave mixing (FWM) to give rise to an idler wave in the 1900$2100 \mathrm{~nm}$ range. For a pulsed pump wave, an idler wave with similar temporal characteristic is obtained; any reconfigurability features of the initial pulsed source are instantaneously copied onto the idler. However since such wideband FWM remains relatively inefficient, mainly due to dispersion fluctuations along the fiber, the average power 
obtained in the SWIR after the HNLF is generally low, even in pulsed pumping regime [2]. To overcome such limitation we previously suggested directly inserting a segment of TDF after the parametric converter [8]. The effect is twofold; first the TDF acts as a filter such that the O-band signal and L-band pump are absorbed by the $\mathrm{Tm}^{3+}$ ions, advantageously removing this undesired high power pump wave at the output of the source; second, the de-excitation of the ${ }^{3} \mathrm{~F}_{4}{ }^{3} \mathrm{H}_{6}$ transition strongly amplifies the idler located in the $2 \mu \mathrm{m}$ region [8]. The schematic principle is plotted in Fig. 1.

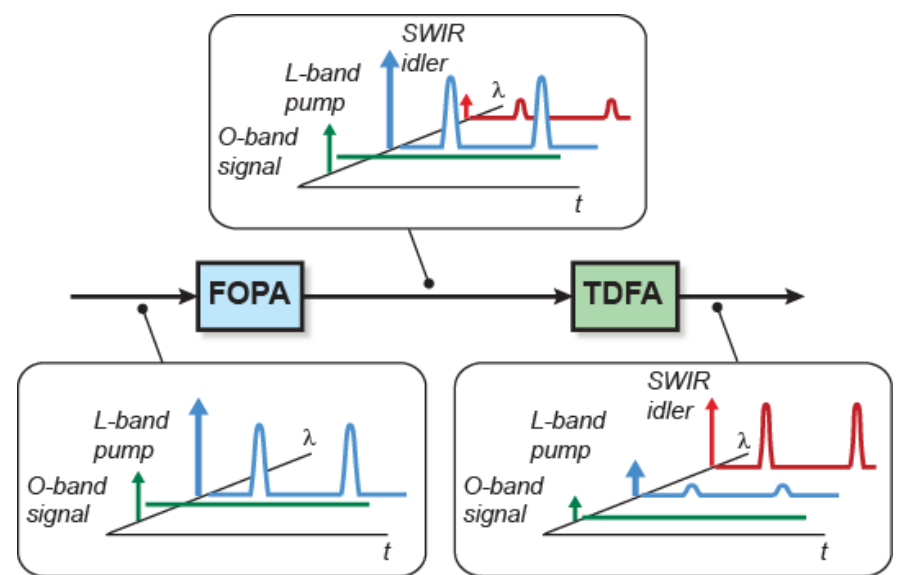

Fig. 1. Schematic of the pulsed idler generation and amplification in each stage. FOPA: fiber optical parametric amplifier, TDFA: thulium-doped fiber amplifier.

In our previous work on thulium-assisted parametric conversion, all interacting waves were continuous, yielding a continuous-wave $(\mathrm{CW})$ idler reaching a power of more than $700 \mathrm{~mW}$ over a wide wavelength range. An advantage of such a cavity-less generation scheme is that any modulation format on the pump or on the signal can in theory be reproduced on the idler [10], [11]. In practice a combination of linear and nonlinear effects along the propagation (dispersion, walk-off, self-phase modulation (SPM)) prevent perfect replication, especially if the interaction waveguide is long and the pulse duration is short. An optimization of the mixing fiber is therefore required in order to accommodate a pulsed pump featuring a repetition rate in the $\mathrm{GHz}$ range with picosecond pulse duration coupled with a $\mathrm{CW}$ O-band signal. In order to obtain a versatile SWIR pulsed source in terms of central wavelength, repetition rate or pulse width the generation of the L-band seed must also be malleable in terms of repetition rate and pulse duration. Moreover, tunability of the pump central wavelength is an interesting feature since parametric conversion in a HNLF requires some fine adjustment around the zero-dispersion wavelength (ZDW) in order to reach the most efficient conversion to the desired frequency [12]. Finally it is advantageous to keep the pump spectrally narrow (transform limited in the best case) since the pump spectral width is transferred to the idler along the propagation following the energy conservation principle [13].

For all these reasons and given the targeted frequencies, the L-band pulse train is shaped using a cascaded intensity modulators architecture [14]. The basic method consists of processing a CW laser through two successive Mach-Zehnder modulators (MZM), one being driven at a frequency $v$ and the second one at a frequency $3 \mathrm{v}$. When a proper bias and radiofrequency (RF) power are applied to the modulators a rectangular frequency comb of 9 lines spanning $9 v$ is synthesized. Provided that the two RF sources are synchronized, the rectangular spectrum corresponds temporally to a train of sinc-shaped pulses at a repetition rate of $v$ and peak-to-zero crossing of $1 / 9 v$ seconds. Such method allows for the required flexibility: the repetition rate and the duty cycle can be modified easily by controlling the RF driving signals or by adding a third stage as will be discussed in section IV. The wavelength can be adjusted to any wavelength within the operating range of the modulators. Last but not least, this method relies solely on standard RF and telecom components.

\section{B. Experimental setup}

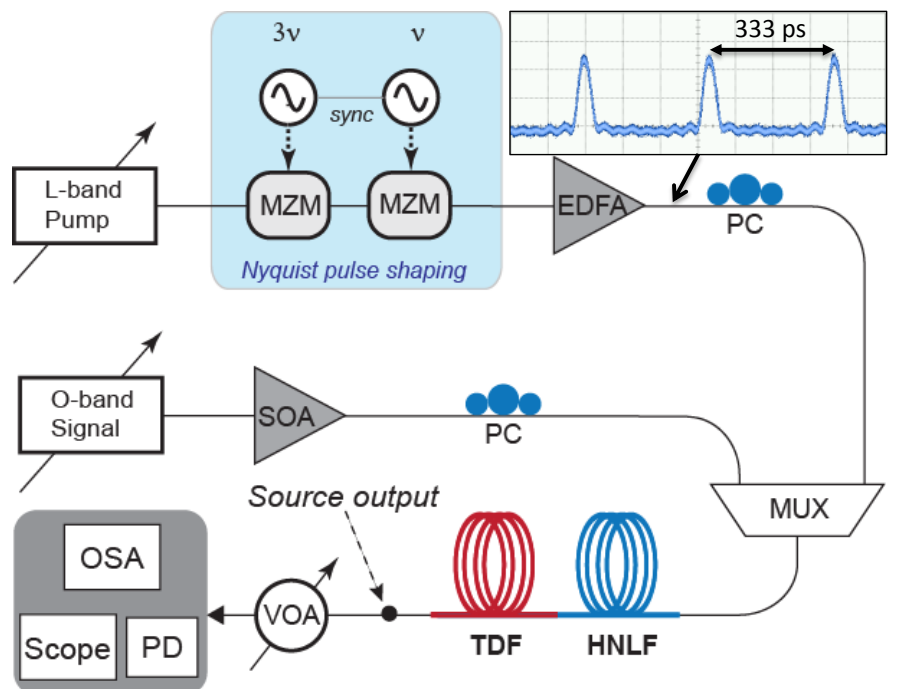

Fig.2. Experimental setup. MZM: Mach-Zehnder modulator, SOA: semiconductor optical amplifier, PC: polarization controller, EDFA: erbiumdoped fiber amplifier, MUX: 1310-1550nm wavelength multiplexer, VOA: variable optical attenuator, OSA: optical spectrum analyzer, PD: $2 \mu \mathrm{m}$ photodetector. Inset: Pump pulse waveform at the HNLF input (recorded with a $50 \mathrm{GHz}$ detector) at a repetition rate of $3 \mathrm{GHz}$.

The experimental setup is sketched on Fig. 2. The pump is shaped as previously described. A tunable continuous-wave and narrow linewidth $(100 \mathrm{kHz})$ external cavity laser is sent through the two intensity modulators before being amplified to an average power between 1 and $2.5 \mathrm{~W}$. For this demonstration, the repetition rate could be tuned to $v=2 \mathrm{GHz}$, $3 \mathrm{GHz}$ or $5 \mathrm{GHz}$. The repetition rate can however be easily scales to higher values. The pulse train at $3 \mathrm{GHz}$ is shown in the inset of Fig. 2. The amplified spontaneous emission (ASE) from the booster is not filtered before the HNLF and is recycled in the next stage to pump the TDF. The boosted pump is then coupled to an amplified tunable CW O-band laser $(13 \mathrm{dBm})$ before being launched into the HNLF and the TDF.

For pulsed operation up to $5 \mathrm{GHz}$, the length of the HNLF was optimized at $30 \mathrm{~m}$. The HNLF from Sumitomo Electric 
features a nonlinear coefficient $\gamma=14 \mathrm{~W}^{-1} \mathrm{~km}^{-1}$, an average ZDW of $1569.05 \mathrm{~nm}$, a third order dispersion $\beta_{3}=4.6 \cdot 10^{-2}$ $\mathrm{ps}^{3} / \mathrm{km}$ and a fourth order dispersion assumed negative in average. Dispersion fluctuations originating from geometrical and doping fluctuations along the waveguide have a large impact on the evaluation of these parameters and on the efficiency of FWM when the pump-idler detuning is of the order of $40 \mathrm{THz}$ [15]-[18]. The behavior of this fiber is further analyzed in the next section. The HNLF is followed by a commercial TDF (OFS TmDF200) of either $11.5 \mathrm{~m}$ or $17.5 \mathrm{~m}$ depending on the targeted output wavelength. An optical attenuator is positioned at the output of the source to control the power sent to the measurement instruments; an optical spectrum analyzer (OSA) and an extended InGaAs $2 \mu \mathrm{m}$ photodiode having a $22 \mathrm{GHz}$ bandwidth. The wavelength dependence of the attenuator is taken into account when power levels or power ratios are computed from the optical spectra. Additionally a thermal power sensor is also used for absolute measurements after the HNLF or the TDF.

\section{RESULTS AND DISCUSSION}

\section{A. Parametric converter behavior}

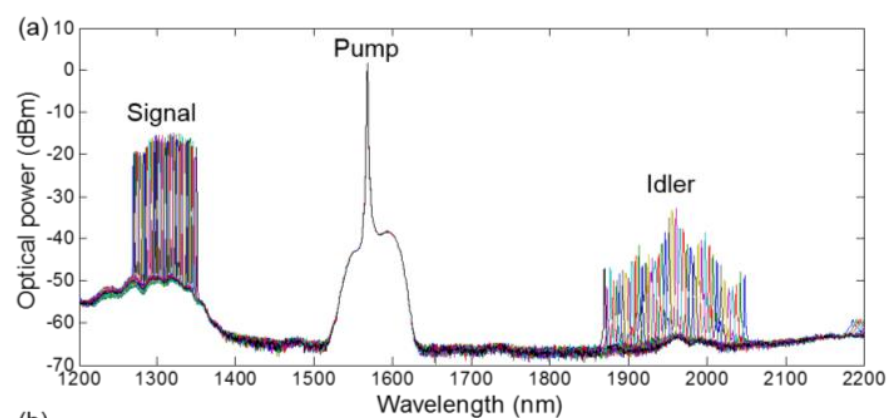

(b)

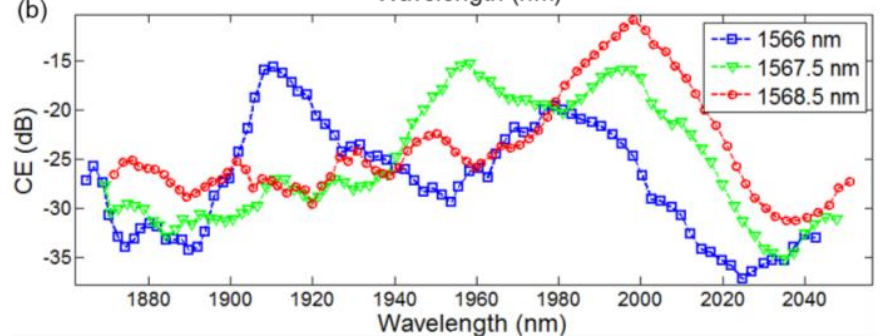

Fig. 3. (a) Superimposed FWM spectra after the HNLF for a pump wavelength of $1567.5 \mathrm{~nm}$ and a signal swept over the O-band (resolution $1 \mathrm{~nm}$ ). (b) Conversion efficiency after the HNLF as a function of the idler wavelength and for the following pump wavelengths: $1566 \mathrm{~nm}, 1567.5 \mathrm{~nm}$ and $1568.5 \mathrm{~nm}$.

In order to optimize the overall performance of the 2 micron source, the parametric conversion in the HNLF is characterized. Due to phase matching considerations, slight adjustments to the pump wavelength are necessary in order to maximize the idler's intensity at any selected wavelength. Indeed even if the TDF stage can compensate for a low idler power at the HNLF output, the optical signal-to-noise ratio (OSNR) after Thulium amplification will be low if the TDF is not seeded with a sufficiently powerful idler. The protocol is the following: for each pump wavelength, the O-band signal is swept and the idler powers are recorded. The results were recorded for a $2 \mathrm{GHz}$ repetition rate, a pump launch power of
$1.7 \mathrm{~W}$ (in average) and are summarized in Fig. 3. Superimposed spectra for a $1567.5 \mathrm{~nm}$ pump are plotted in Fig. 3(a) while the conversion efficiency (CE) as a function of idler wavelength for three pump positions is shown in Fig. 3(b). The spectra are shown as they appeared on the OSA after the attenuator. The $\mathrm{CE}$ is computed as the ratio between the idler "effective power" (pulse energy over pulse duration) and the signal average power, both at the output of the HNLF, taking the wavelength-dependent attenuation function of the attenuator into account. The approximation is valid since the low efficiency of the FWM process yields a negligible change in the signal power. As expected the CE spectral peak evolves with the pumping wavelength, but the spectra also features multiple peaks shifting without apparent correlation, contrarily to what happens in a dispersion stabilized fiber [19]. In first approximation the multi-peaks effect of the $\mathrm{CE}$ can be ascribed to dispersion variations along the waveguide having a long correlation length whereas the peaks broadening is attributed to the short-correlation length fluctuations. This effect is however hard to model and we limited ourselves to an empirical analysis in order to determine which pumping wavelength is optimal when targeting a given output wavelength for the laser. Two cases are studied in this article: the SWIR output around $1958 \mathrm{~nm}$ (pump at $1567.5 \mathrm{~nm}$ ) and the SWIR output around $2001 \mathrm{~nm}$ (pump at $1568.5 \mathrm{~nm}$ ). As it can be seen on Fig. 3(b), these configurations should yield optimized idler powers after the HNLF.

\section{B. Overall source behavior}

For the characterization of the source at a $1958 \mathrm{~nm}$ central wavelength, an $11.5 \mathrm{~m}$ TDF is appended at the HNLF output. The use of a relatively long doped fiber is necessary to redshift the peak of the gain spectrum toward $1958 \mathrm{~nm}$ and avoid detrimental effects such as spontaneous lasing [17] or OSNR degradation due to ASE. Spectra and pulse waveforms recorded right after the TDF are shown on Fig. 4. The spectra before and after the TDF for a $2 \mathrm{GHz}$ repetition rate and a $1.7 \mathrm{~W}$ of pump power (at the HNLF input) are plotted in Fig. 4(a). The $1958 \mathrm{~nm}$ idler undergoes an amplification of $30 \mathrm{~dB}$ through the TDF while the L-band pump is entirely absorbed and the signal at $1310 \mathrm{~nm}$ strongly attenuated $(20 \mathrm{~dB})$. No filter is used after the TDF to remove ASE or to select the wavelength. Adjustment of the emission peak and proper idler seed power are sufficient to guarantee a good OSNR (33 dB considering the peak to pedestal amplitude) at the desired wavelength after the amplifier.

The average power in SWIR at the source output in this configuration is measured to be $160 \mathrm{~mW}$, independently of repetition rate. The waveforms at $2 \mathrm{GHz}, 3 \mathrm{GHz}$ and $5 \mathrm{GHz}$ repetition rate as seen in Fig. 4(b-d), respectively, indicate the generation of clean pulse trains, even in the absence of ASE filtering device before the detector. From the oscilloscope, the pulse duration in the three cases is $45 \mathrm{ps}, 36 \mathrm{ps}$ and $33 \mathrm{ps}$. However, except for the $2 \mathrm{GHz}$ case, the pulse bandwidth exceeds the one of the photodiode (as specified by the manufacturer) such that the value for higher speeds is likely to be limited by the photodiode impulse response. For the $2 \mathrm{GHz}$ 
case, given the pulse duration, a pulse peak power of approximately $620 \mathrm{~mW}$ is retrieved. This value is expected to be similar in all three cases as the initial duty cycle is identical. Finally the idler spectra at the three rates are shown in Fig. 4(e) with, as expected, a slight broadening when $v$ is increased and consequently when the pump pulses are shortened. We ascribed this broadening to the SPM undergone by the pump, which becomes more pronounced as the pulse rise time is reduced. The spectral broadening is then transferred to the idler during its generation, as discussed in section II. Cross-phase modulation, which exhibits a similar physics, is also likely to play a role here. Nonetheless, we believe that the idler is not powerful enough to be directly broadened by SPM through the HNLF, although it may happen in the TDF stage afterward [20].
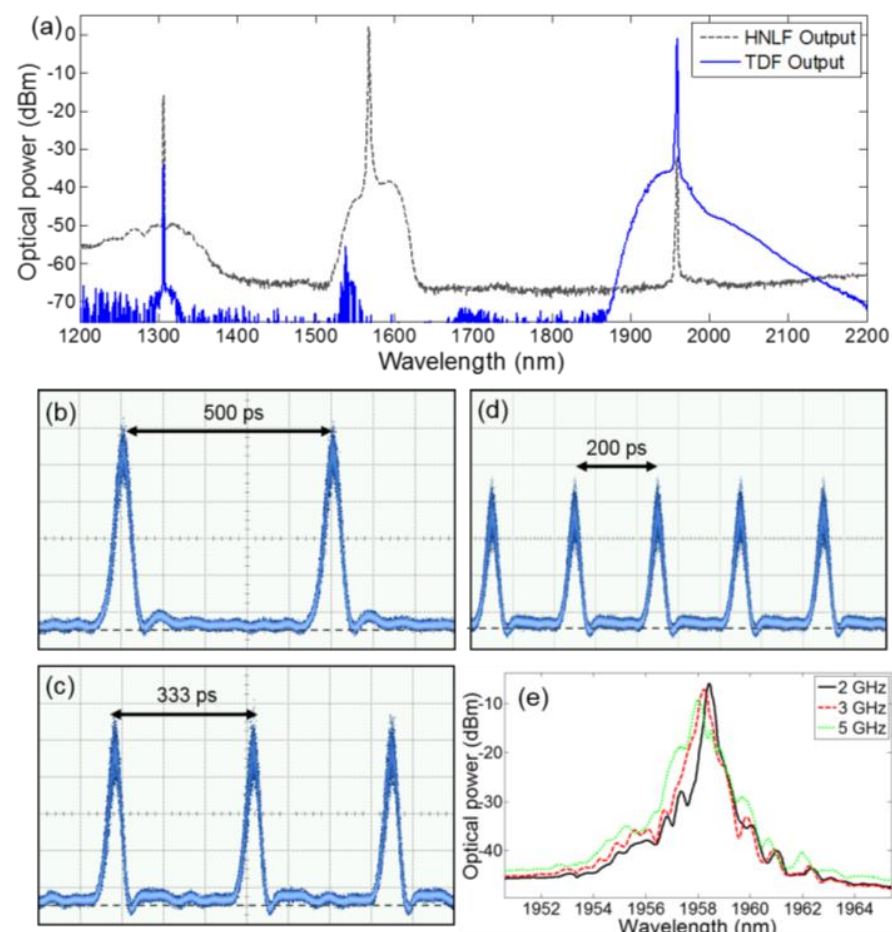

Fig. 4. (a) Spectra at the output of the HNLF and TDF for the $1958 \mathrm{~nm}$ idler configuration (resolution $1 \mathrm{~nm}$ ). Pulse train recorded at the output of the source for a repetition rate of (b) $2 \mathrm{GHz}$, (c) $3 \mathrm{GHz}$ and (d) $5 \mathrm{GHz}$. The dashed lines show the $0 \mathrm{~V}$ level. (e) Spectra of the idler at various repetition rate (resolution $0.1 \mathrm{~nm}$ ).

Similar characterization was performed for the generation of a $2001 \mathrm{~nm}$ idler. In this configuration the pump was positioned at $1568.5 \mathrm{~nm}$ and a longer piece of TDF was used to further red-shift the peak of the Thulium gain. In this case however, as the pumping power is limited by the previous stage and as the $\mathrm{Tm}^{3+}$ ions feature some absorption at $2 \mu \mathrm{m}$ when they are not excited, a tradeoff must be found. A length of $17.5 \mathrm{~m}$ was selected as the best compromise in spite of the presence of a slight spontaneous lasing around $1919 \mathrm{~nm}$ (see Fig. 4(a)). It should be noted that even if the CE peaks at $-11 \mathrm{~dB}$ in this configuration (see Fig. 3(c)), the O-band semiconductor amplifier is quite weak at the corresponding signal wavelength $(1290 \mathrm{~nm})$, and the subsequent idler absolute power drop leads to a less effective seeding of the
TDF stage. We measure a $9 \mathrm{~dB}$ difference between the idler generated at $1958 \mathrm{~nm}$ and the $2001 \mathrm{~nm}$ one. Despite the still $30 \mathrm{~dB}$ amplification, the OSNR is therefore slightly degraded, translating to the addition of noise on the pulse train when the modulation speed is increased as can be seen in Fig. 5(b-d), in contrast with the previous results. This effect is further magnified due to the cutoff-frequency of the detector and leads to a poor waveform at $5 \mathrm{GHz}$. While a stronger signal source or a SWIR filter would be the most straightforward solutions, a dual-stage, long-wave amplification architecture would also help suppressing the ASE peak at $1950 \mathrm{~nm}$ [8], [21]. Finally, in such configuration the output average power is measured to be $37 \mathrm{~mW}$, for a pulse peak power of approximately $142 \mathrm{~mW}$ at $2 \mathrm{GHz}$, while the idler spectra show a similar behavior as to the $1958 \mathrm{~nm}$ case (Fig. 5(e)).
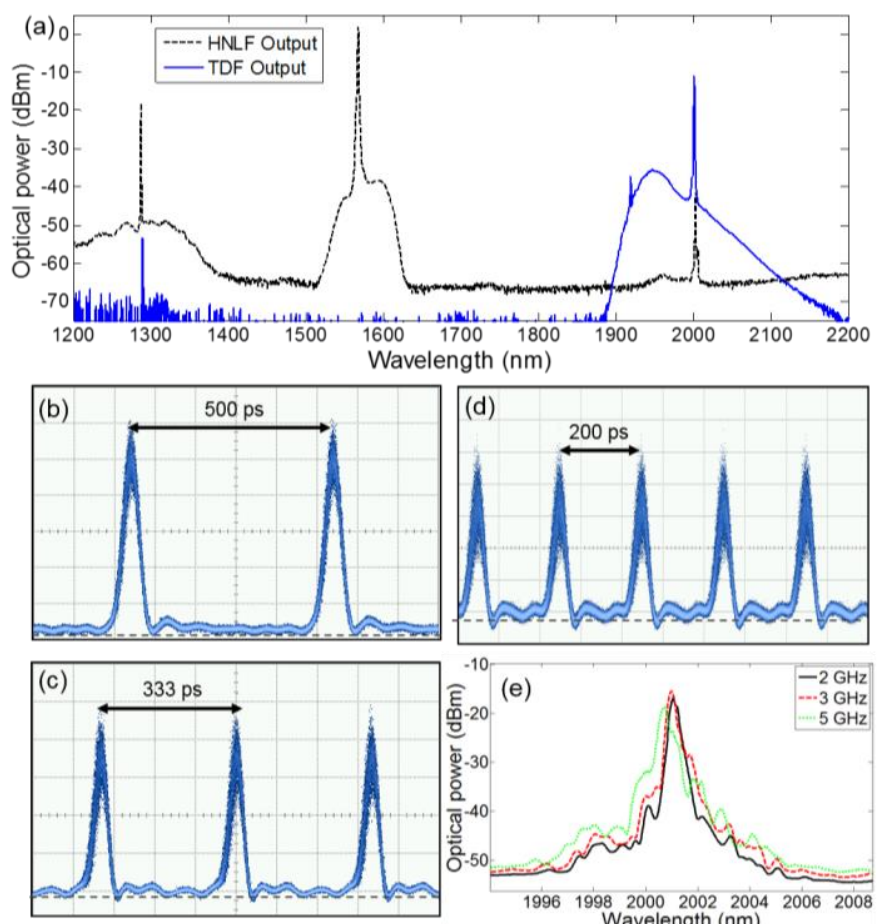

Fig 5. (a) Spectra at the output of the HNLF and TDF for the $2001 \mathrm{~nm}$ idler configuration (resolution $1 \mathrm{~nm}$ ). Pulse train recorded at the output of the source for a repetition rate of (b) $2 \mathrm{GHz}$, (c) $3 \mathrm{GHz}$ and (d) $5 \mathrm{GHz}$. The dashed lines show the $0 \mathrm{~V}$ level. (e) Spectra of the idler at various repetition rate (resolution $0.1 \mathrm{~nm}$ ).

In both cases presented, the CE curves shown on Fig. 3(c) demonstrate that the pulse central wavelength can be tuned over several $\mathrm{nm}$ around the peak values located at 1960 and $2000 \mathrm{~nm}$, only by sweeping the O-band signal wavelength. For a larger SWIR wavelength change, the pump and the TDF amplifier have to be reconfigured as well. Overall the $\mathrm{CE}$ curves suggest that the source could operate between $1900 \mathrm{~nm}$ and $2000 \mathrm{~nm}$ seamlessly.

\section{Data transmission in SWIR}

As previously mentioned an advantage of such cavity-less pulsed source is the possibility to easily modify the architecture to operate as a data transmitter. To strengthen this claim, we conducted an experiment to generate wavelength 
tunable high speed return-to-zero on-off keying (RZ-OOK) data streams in the SWIR band. The OOK modulation is added on the pump pulse train by inserting a data modulator driven by a $2^{31}-1$ pseudo-random bit sequence after the pulse shaping. The experiment was conducted for $3 \mathrm{~Gb} / \mathrm{s}$ data generation and for a pump set at $1967.5 \mathrm{~nm}$. Other settings could have easily been implemented. To additionally verify the wavelength tunability of the source, the O-band signal is swept between $1297.5 \mathrm{~nm}$ and $1320 \mathrm{~nm}$ leading to the generation of a RZ-OOK modulated idler in the range 1929 $1980 \mathrm{~nm}$. Within this range, we recorded the output OSNR of the signal and the bit error rate (BER). The SWIR bit sequence received on the photodiode is analyzed by an error-detector. During the procedure, the idler spectral peak power is kept constant at the photodiode input for all wavelengths at about $-7 \mathrm{dBm}$. The results are summarized in Fig. 6. The eye patterns observed on the oscilloscope for two of the wavelength, $1940 \mathrm{~nm}$ and $1979 \mathrm{~nm}$, are shown in Fig. 6(a-b). Other wavelengths exhibited similar characteristics with a wide-open eye and a high extinction ratio. At such input power, the data conversion had a BER less than $10^{-9}$ over the whole tested wavelength range, in spite of some OSNR variations due to the variation in $\mathrm{CE}$ at the output of the HNLF. Even with the highest ASE level (such as at $1929 \mathrm{~nm}$ ), the eye pattern is still wide open. As a simple observation, the noise appears to simply offset the entire waveform, adding a continuous component but leaving it clean for data carrying. It proves that a large degree of choice in the output wavelength is permitted without modifying the pump wavelength.
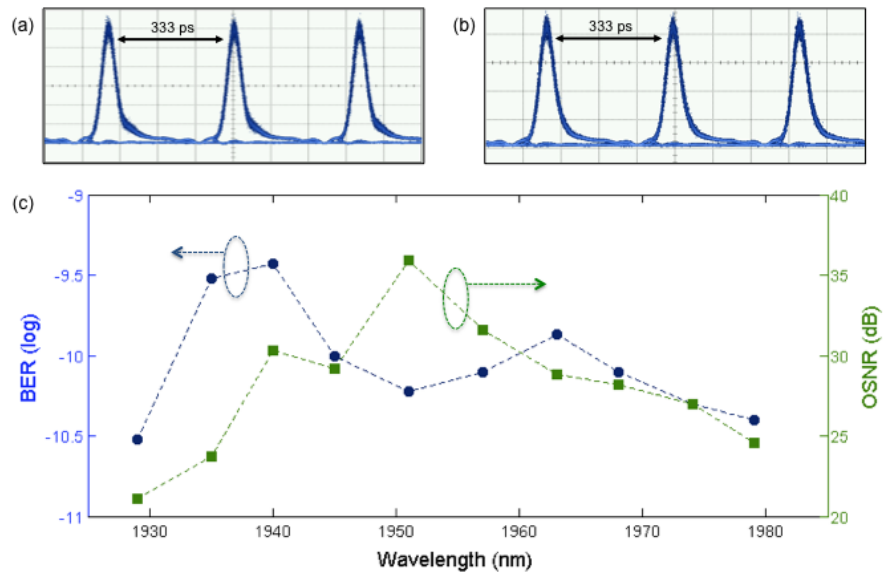

Fig. 6. Eye patterns of the modulated idlers at (a) $1940 \mathrm{~nm}$ and (b) $1979 \mathrm{~nm}$. (c) BER (circles, left axis) and OSNR (squares, right axis) recorded for each tested wavelengths at a bit rate of $3 \mathrm{~Gb} / \mathrm{s}$.

\section{CONCLUSION}

In summary, we presented an all-fiber picosecond pulsed source capable of $\mathrm{GHz}$ repetition rate in the $2 \mu \mathrm{m}$ band. It is based on FWM between a CW O-band signal, a pulsed L-band pump resulting in a pulsed SWIR idler. The latter is then amplified in a TDF pumped by the L-band wave in an energy efficient scheme relying only on standard telecom components. Thanks to the versatility of the Nyquist pulse carver, the repetition rate can be electrically controlled at will. A good replication of the original pulse train is maintained at a rate up to $5 \mathrm{GHz}$ in the $1958 \mathrm{~nm}$ output configuration, with pulse duration of tens of picosesonds. We demonstrate the source operation around $1958 \mathrm{~nm}$ and $2001 \mathrm{~nm}$. Even though the wavelength range accessible by sweeping only the signal is narrower than in previous experiments using a longer HNLF [22] due to the reduced amount of cumulated dispersion fluctuations, the entire range between $1900 \mathrm{~nm}$ and $2000 \mathrm{~nm}$ is accessible with a proper adjustment of the pump wavelength. For the source to operate at longer wavelengths $(>2 \mu \mathrm{m})$, a powerful CW O-band laser and a long-band TDF amplifier configuration are necessary. The laser also proves able to output a RZ-OOK data sequence in SWIR as is shown at $3 \mathrm{~Gb} / \mathrm{s}$ rate. This feature is necessary for SWIR free-space communications and could be used to realize a fast modulation of a lidar or spectrometer probing beam. To further improve the quality of the source for such applications it is possible to insert a bandpass filter at its output to remove the ASE.

Moreover, enhancements that can be easily implemented with the Nyquist pulse carver scheme theoretically allow for a decoupled control of the pulse duration and rep rate. On the one hand it has been shown that seeding one of the modulators with two or more properly selected RF tones allows for the densification of the rectangular frequency comb while preserving its linewidth [14]. This translates into reducing the pump repetition rate while keeping the same pulse duration and should impact the source output in the same way. On the other hand it is possible to keep the same spectral line spacing and broaden it with a third modulator or via FWM in a HNLF [23] before boosting it. This means that the pump pulse duration would be decreased at constant repetition rate. In both cases an augmentation of the pump peak will be obtained after the booster. As a result the CE should be increased through the HNLF, enabling the use of a shorter fiber. The main advantage of reducing the interacting length without sacrificing conversion efficiency, is the possible increase of repetition rate (over $5 \mathrm{GHz}$ ) and/or decrease of the pulse duration (below $30 \mathrm{ps}$ ). As the nonlinear medium gets shorter, linear and nonlinear propagation phenomena's impact is reduced. In long waveguides and for ps pulses, SPM broadens dramatically the pump spectrum, preventing a good idler linewidth in SWIR. Additionally the high dispersion at $2 \mu \mathrm{m}$ will chirp the generated pulses along the propagation whereas a group velocity mismatch between the L-band and the SWIR band will create a walk-off that broadens them during their generation. All these adverse effects are quenched when the fiber is kept short, potentially allowing increasing the repetition rate up to $10 \mathrm{GHz}$ or more.

\section{ACKNOWLEDGMENT}

The authors thank Sumitomo Electric Industries for providing the HNLF used in this study.

\section{REFERENCES}

[1] J. Geng, and S. Jiang, "Fiber Lasers: The $2 \mu \mathrm{m}$ Market Heats Up," Opt. Photonics News, vol. 25, no. 7, pp. 34-41, Jul. 2014. 
[2] S. Moro, A. Danicic, N. Alic, N. G. Usechak, and S. Radic, "Widelytunable parametric short-wave infrared transmitter for $\mathrm{CO} 2$ trace detection," Opt. Express, vol. 19, no. 9, pp. 8173-8178, Apr. 2011.

[3] Z. Liu, Y. Chen; Z. Li, B. Kelly, R. Phelan, J. O'Carroll, T. Bradley, J. P. Wooler, N. V. Wheeler, A. M. Heidt, T. Richter, C. Schubert, M. Becker, F. Poletti, M. N. Petrovich, S.-U. Alam, D. J. Richardson and, R. Slavik, "High-Capacity Directly Modulated Optical Transmitter for 2$\mu \mathrm{m}$ Spectral Region," J. Lightw. Technol., vol. 33, no.7, pp. 1373-1379, Apr. 2015.

[4] M. Zhang, E. J. R. Kelleher, T. H. Runcorn, V. M. Mashinsky, O. I. Medvedkov, E. M. Dianov, D. Popa, S. Milana, T. Hasan, Z. Sun, F. Bonaccorso, Z. Jiang, E. Flahaut, B. H. Chapman, A. C. Ferrari, S. V. Popov, and J. R. Taylor, "Mid-infrared Raman-soliton continuum pumped by a nanotube-mode-locked sub-picosecond Tm-doped MOPFA," Opt. Express, vol. 21, pp. 23261-23271, Sept. 2013.

[5] X. Liu, B. Kuyken, G. Roelkens, R. Baets, R. M. Osgood Jr, and W. M. J. Green, "Bridging the mid-infrared-to-telecom gap with silicon nanophotonic spectral translation," Nature Photon., vol. 6, no. 10, pp. 667-671, Sep. 2012.

[6] Q. Fang, K. Kieu, and N. Peyghambarian, "An All-Fiber 2- $\mu \mathrm{m}$ Wavelength-Tunable Mode-Locked Laser," IEEE Photon. Technol. Lett., vol. 22, no. 22, pp. 1656-1658, Nov. 2010.

[7] J. M. Chavez Boggio, S. Moro, B. P.-P. Kuo, N. Alic, B. Stossel, and S Radic, "Tunable Parametric All-Fiber Short-Wavelength IR Transmitter," J. Lightwave Technol., vol. 28, no. 4, pp. 443-447, Feb. 2010.

[8] A. Billat, S. Cordette, Y.-P. Tseng, S. Kharitonov, and C.-S. Brès, "High-power parametric conversion from near-infrared to short-wave infrared," Opt. Express, vol. 22, no. 12, pp.14341-14347, Jun. 2014.

[9] S. D. Agger and J. H. Povlsen, "Emission and absorption cross section of thulium doped silica fibers," Opt. Express, vol. 14, no. 1, pp. 50-57, Jan. 2006.

[10] F. Gholami, S. Zlatanovic, E. Myslivets, S. Moro, B. P.-P. Kuo, C.-S. Brès, A. O. J. Wiberg, N. Alic, and S. Radic, "10Gbps Parametric ShortWave Infrared Transmitter," presented at the Opt. Fiber Commun. Conf., Los Angeles, 2011, paper OThC6.

[11] F. Gholami, B. P.-P. Kuo, S. Zlatanovic, N. Alic, and S. Radic, "Phasepreserving parametric wavelength conversion to SWIR band in highly nonlinear dispersion stabilized fiber," Opt. Express, vol. 21, no. 9, pp. 11415-11424, May 2013.

[12] B. P.-P. Kuo, N. Alic, P. F. Wysocki, and S. Radic, "Simultaneous Wavelength-Swept Generation in NIR and SWIR Bands Over Combined 329-nm Band Using Swept-Pump Fiber Optical Parametric Oscillator," J. Lightwave Technol., vol. 29, no. 4, pp. 410-416, Feb. 2011.

[13] H. Min-Chen, M. E. Marhic, K. Y. K. Wong, and L. G. Kazovsky, "Narrow-linewidth idler generation in fiber four-wave mixing and parametric amplification by dithering two pumps in opposition of phase," J. Lightw. Technol., vol.20, no. 3, pp. 469-476, Mar. 2002.

[14] M. A. Soto, M. Alem, M. A. Shoaie, A. Vedadi, C.-S. Brès, L. Thévenaz, and T. Schneider, "Optical sinc-shaped Nyquist pulses of exceptional quality," Nat. Commun., vol. 4, no. 298, pp. 2-11, Dec. 2013.

[15] M. Farahmand, and M. de Sterke, "Parametric amplification in presence of dispersion fluctuations," Opt. Express, vol. 12, no. 1, pp. 136-142, Dec. 2003.

[16] J. M. Chávez Boggio, and H. L. Fragnito, "Simple four-wave-mixingbased method for measuring the ratio between the third- and fourthorder dispersion in optical fibers," J. Opt. Soc. Am. B, vol. 24, no. 9, pp. 2046-2053, Sep. 2007.

[17] A. Billat, S. Cordette, and C.-S. Brès, "Broadly tunable source around $2050 \mathrm{~nm}$ based on wideband parametric conversion and thuliumholmium amplification cascade," Opt. Express, vol. 22, no. 22, pp. 26635-26641, Oct. 2014.

[18] J. S. Y. Chen, S. G. Murdoch, R. Leonhardt, and J. D. Harvey, "Effect of dispersion fluctuations on widely tunable optical parametric amplification in photonic crystal fibers," Opt. Express, vol. 14, no. 20, pp. 9491-9501, Oct. 2006.

[19] B. P.-P. Kuo, M. Hirano, and S. Radic, "Continuous-wave, shortwavelength infrared mixer using dispersion-stabilized highly-nonlinear fiber," Opt. Express, vol. 20, no. 16, pp. 18422-18431, Jul. 2012.

[20] S. Kharitonov, A. Billat, L. Zulliger, S. Cordette, A. Vedadi, and C.-S. Brès, "Kerr nonlinearity of Thulium-doped fiber near $2 \mu \mathrm{m}$," presented at CLEO, San Jose, 2015, paper JTu5A.31.
[21] Z. Li, A. M. Heidt, J. M. O. Daniel, Y. Jung, S. U. Alam, and D. J. Richardson, "Thulium-doped fiber amplifier for optical communications at $2 \mu \mathrm{m}$," Opt. Express, vol. 21, no. 8, pp. 9289-9297, Apr. 2013.

[22] A. Billat, S. Cordette, and C.-S. Brès, "2 microns all-fiber picosecond pulse source with gigahertz repetition rate," presented at CLEO EuropeEQEC 2015, Munich, 2015, paper CD-1.6.

[23] S. Cordette, A. Vedadi, M. A. Shoaie, and C.-S. Brès, "Bandwidth and repetition rate programmable Nyquist sinc-shaped pulse train source based on intensity modulators and four-wave mixing," Opt. Lett., vol. 39, no. 23, pp. 6668-6671, Dec. 2014.

Adrien Billat was born in Grenoble, France, in 1989 and received the Diplôme d'Ingénieur from the Ecole Supérieure d'Electricité (Supélec), Paris, France, and the M.Sc. degree from the Ecole Polytechnique Fédérale de Lausanne (EPFL), Lausanne, Switzerland, both in 2013 and in Electrical Engineering. He is currently working toward the Ph.D. degree in Electrical Engineering from EPFL as well.

His current research interests are very broadband fiber optical parametric converters, thulium-doped fiber lasers and amplifiers and silicon-based nonlinear photonics.

Steevy Cordette (S'06, M'10) received the M.Sc. degree in electrical engineering from the Higher School of Electrical Engineering (ESIGELEC), Rouen, France, and the M.Sc degree in Electromagnetism and Optoelectronics from the National Higher School of Aeronautics and Space (Supaero), Toulouse, France, in 2006. He obtained his Ph.D. degree in electrical engineering from the National Higher School of Telecom (Telecom ParisTech), Paris, France, in 2010.

After 2 years as a consultant in the Telecom industry, he joined, as research scientist, the Photonic Systems Laboratory (PHOSL) at the Ecole Polytechnique Fédérale de Lausanne (EPFL), in Switzerland. His main research interests include nonlinear optical phenomena, all-optical signal processing, light generation, and optical communications.

Dr. Cordette has published more than 20 scientific papers in journals and international conferences, and holds 3 patents.

Camille-Sophie Brès (S'02, M'07) is an assistant professor at Ecole Polytechnique Fédérale de Lausanne (EPFL), in Switzerland. She obtained her B.Eng. with honors in electrical engineering from McGill University in 2002 and her Ph.D. from Princeton University in 2007. Prior to joining EPFL in 2011, she was a research scientist at the University of California San Diego. Her research interests are in the areas of all optical signal processing based on nonlinear effects waveguides, Mid IR light generation and optical communications. Prof. Brès has published over 100 articles in peer reviewed journals and international conferences. She currently serves on the technical program of the Optical Fiber Communication conference (OFC) and the European Conference on Optical Communication (ECOC) committees. She is the recipient of an ERC starting grant in 2012 and of the European Optical Society LIGHT2015 Young Women in Photonics Special Recognition award. 